\title{
Usefulness of Topical Interferon Alpha-2b Eye Drop as an Adjunctive Therapy Following Surgical Resection in Ocular Surface Squamous Neoplasia
}

\author{
RINA KANAYA, SATORU KASE, KAN ISHIJIMA and SUSUMU ISHIDA \\ Department of Ophthalmology, Faculty of Medicine and Graduate School of Medicine, \\ Hokkaido University, Sapporo, Japan
}

\begin{abstract}
Aim: To report the clinical course of patients with ocular surface squamous neoplasia treated with topical interferon $\alpha-2 b(I F N \alpha-2 b)$ after local excision of the tumor. Patients and Methods: This study enrolled four consecutive Japanese patients comprising one eye with conjunctival carcinoma in situ, and three eyes with squamous cell carcinoma (SCC) diagnosed histopathologically. All of them initially visited Hokkaido University Hospital in 2016. After resecting the tumor tissues, topical IFN $\alpha$-2b eye drops were given to the eye four times a day. This study retrospectively analyzed the ophthalmological and imaging findings before and after the surgery, based on the patients' medical records. Results: The mean follow-up duration was 37.8 months. All cases were males, and the mean age was 62.3 years. The tumor was located in the bulbar conjunctiva in three cases. Local pedunculated rotation flap, and free conjunctival flap were performed in one and two patients, respectively. In one case with SCC, the tumor involved the lower palpebral conjunctiva, already invading into the orbit at the first visit. The patient underwent extended resection of the lower lid, and reconstruction of the posterior lobe of the eyelid with Hughes flap. Cataract surgery was successfully conducted 18 months after tumor resection. All patients remain well without local recurrence or distant metastasis. Conclusion: Topical IFN $\alpha-2 b$ treatment contributed to suppression of tumor recurrence and improvement of quality of vision in patients after local resection of ocular surface squamous neoplasia.
\end{abstract}

This article is freely accessible online.

Correspondence to: Satoru Kase, Department of Ophthalmology, Faculty of Medicine and Graduate School of Medicine , Hokkaido University, N-15, W-7, Kita-ku, Sapporo 060-8638, Japan. Tel: +81 117065944, Fax: +81 117065948, e-mail: kaseron@med.hokudai.ac.jp

Key Words: Conjunctiva, squamous cell carcinoma, interferon, cataract surgery.
Ocular surface squamous neoplasia (OSSN) is the most common non-pigmented tumor of the ocular surface. It comprises a range of dysplasias, corneal-conjunctival carcinoma in situ and invasive squamous cell carcinoma (SCC) (1). The treatment of OSSN comprises local resection of the tumor, adjuvant local chemotherapy, cryotherapy, radiotherapy and enucleation as well as eventual orbital exenteration.

Recently, local chemotherapy has been developed which contributes to preservation of patients' globes containing conjunctival malignancies. Indeed, in order to reduce the risk of recurrence after local resection of the tumors, several topical therapies have been proved effective in OSSN treatment, including mitomycin $\mathrm{C}$, 5-fluorouracil (5-FU), and interferon alpha-2b (IFN $\alpha-2 b)$. Among them, IFN $\alpha-2 b$ plays pleiotropic roles in suppression of tumor growth, thereby leading to activation of host immune system including natural killer cells, macrophages and CD8-positive T-cells (2). Moreover, topical IFN $\alpha-2 b$ is considered a feasible treatment for OSSN because of its minimal toxicity $(3,4)$. A recent comparative study showed that although clinical outcomes seemed to be similar, the frequency of adverse effects is lower in those treated with IFN $\alpha-2 b$ than in 5-FU (5). Therefore, we examined the role of topical IFN $\alpha-2 b$ in conjunctival malignancies; to our knowledge, there are no reports on how topical IFN $\alpha-2 b$ application contributes to the improvement of quality of vision (QOV) in patients with OSSN.

We herein report the clinical course of patients with OSSN treated with IFN $\alpha-2 b$ after local excision of the tumor, and detail the case of a patients with OSSN who safely underwent cataract surgery after this treatment.

\section{Patients and Methods}

This was a retrospective observational study. The ophthalmological and imaging findings were analyzed before and after surgery, based on the patients' medical records. This study enrolled four consecutive Japanese patients comprising one eye with conjunctival carcinoma in situ, and three eyes with SCC 

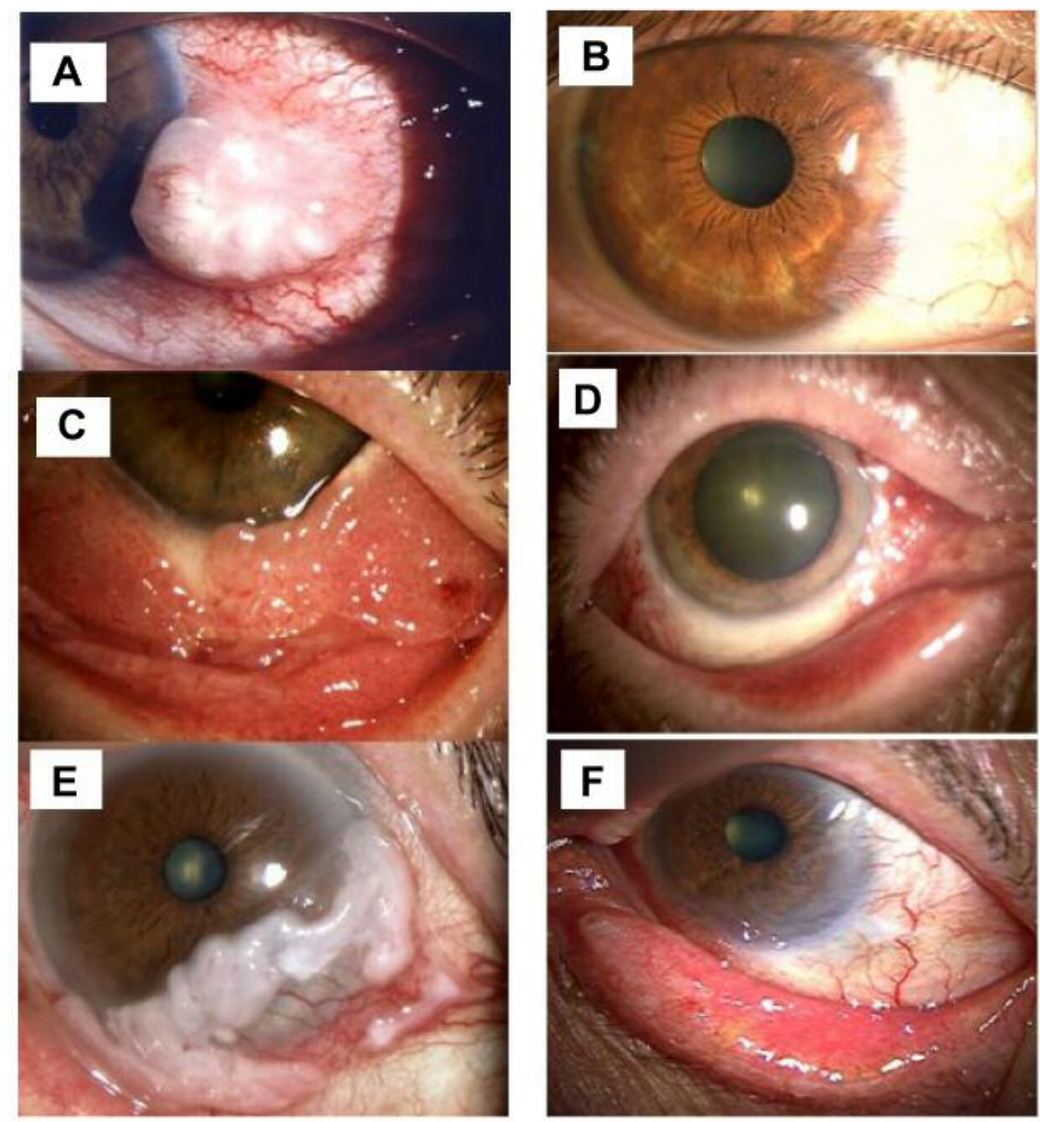

Figure 1. The slit-lamp examinations at their first visit $(A, C, E)$ and after treatment $(B, D, F)$ in cases arising in the bulbar conjunctiva. Case 1. A: A 50-year-old male presented with a whitish nodule located in the bulbar conjunctiva. B: The ocular surface remained smooth 23 months after local resection and adjuvant interferon therapy. Case 2. C: A reddish nodule was apparent in the bulbar conjunctiva of an 80-year-old male. D: After tumor resection, no tumor recurrence was noted 21 months after treatment. Case 3. E: An 81-year-old male presented with a whitish tumor in the bulbar conjunctiva. F: At the 18-month follow-up, there was no local recurrence.

diagnosed histopathologically. All of them initially were diagnosed at Hokkaido University Hospital in 2016. All the patients enrolled in this study were examined by ophthalmological tests including visual acuity, intraocular pressure, slit-lamp examination and fundus examination. The patients also underwent magnetic resonance imaging and systemic computed tomography (CT) as well as positron-emission tomography-CT once a year. After resecting the tumor tissues, topical IFN $\alpha-2 b$ eye drops were given to the eye, at one drop four times a day. Treatment generally lasted for 4 months; if the tumor was suggested to invade the orbit, longer duration was required. The endpoint of use was the result of repeated biopsy, namely being negative for malignancy. The concentration of topical IFN $\alpha$-2b was set to $106 \mathrm{IU} / \mathrm{ml}$ according to a previous report (6). The Institutional Review Board of Hokkaido University Hospital waived the approval of this study as a clinical trial because of a non-invasive study and the limited number of cases examined. No patient with OSSN initially underwent enucleation or orbital exenteration during the designated study period. The presence of distant metastases was assessed by systemic computed tomography 6 to 12 months after surgery.

\section{Results}

Table I summarizes the clinicopathological findings in this study. All of the cases were male, and the mean age was 62.3 years. Figure 1 shows the slit-lamp findings at their first visit for cases 1 to 3 , with OSSN arising in the bulbar conjunctiva (Figure $1 \mathrm{~A}, \mathrm{C}$ and $\mathrm{E}$ ). In case 1, the whitish nodule with marked conjunctival hyperemia was located in the nasal bulbar conjunctiva (Figure 1A). A reddish sessile papillomatous tumor located in the bulbar conjunctiva in case 2 (Figure 1C). In case 3 , the patient presented with a whitish tumor in the bulbar conjunctiva and cornea. In case 4 , the slit-lamp examination showed marked lower eyelid swelling with irregular whitish conjunctival tumor and congestion (Figure 2A). In cases 1 to 3 , the patients were treated with local pedunculated conjunctival flaps and cryotherapy combined with free conjunctival flaps following local resection. Histopathology of the resected tumor tissues revealed malignant tumor cells 

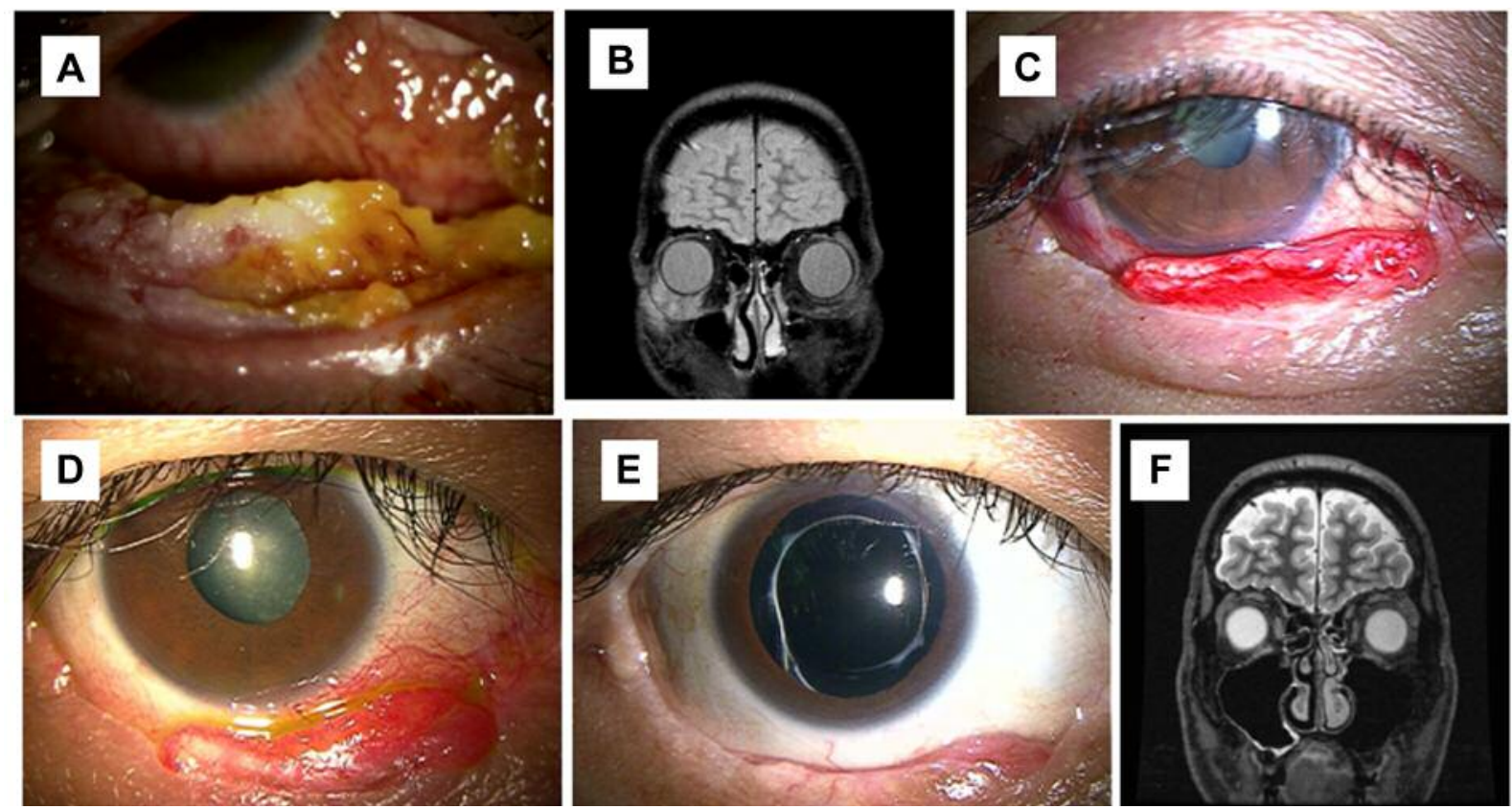

Figure 2. Slit-lamp examination $(A, B, D$ and $E)$ and magnetic resonance imaging $(M R I)(C$ and $F)$ of case 4. A: Slit-lamp examination at the first visit showed an irregular reddish mass located in the lower conjunctiva. B: MRI revealed orbital invasion. C: After eyelid excision followed by Hughes flap reconstruction, the lower eyelid has yet to be epithelialized. D: The reconstructed lower eyelid became epithelialized with smooth ocular surface 12 months after the initial surgery. E: At 24 months after tumor resection, the patient safely underwent cataract surgery. F: Three years after his initial visit, there was no recurrence of the tumor in the orbit.

Table I. Clinicopathological features of patients with ocular surface squamous neoplasia. All patients were males and had no evidence of recurrence or metastasis.

\begin{tabular}{lcccccc}
\hline Case & Age, years & Eye & TNM classification* & Surgical margin & Duration of IFN (months) & Follow-up period (months) \\
\hline 1 & 50 & Right & pT1N0M0 & + & 6 & 40 \\
2 & 80 & Left & pTisN0M0 & + & 2 & 42 \\
3 & 81 & Right & pTisN0M0 & $+/-$ & 3 & 35 \\
4 & 39 & Right & pT2N0M0 & + & 8 & 34 \\
\hline
\end{tabular}

IFN: Interferon- $\alpha-2 b$. *TNM classification was based on AJCC Cancer Staging Manual, 8th edition (10).

differentiating from squamous cells, consistent with OSSN. The tumors were diagnosed as SCC and carcinoma in situ in cases 1, 2, and 4 (Figure 3A, B and D), and case 3 (Figure 3C), respectively. Histopathology of the resected tumor tissues suggested positive surgical margin at the corneal side in all cases. Topical IFN $\alpha-2 b$ was administered to patients for 4 to 12 months. The mean follow-up duration was 37.8 months. All patients are well without local recurrence (Figure 1B, D and F; Figure 2E) or distant metastasis.

\section{Representative Case Report}

The patient was a 39-year-old male complaining of irritation and continuous discharge from his right eye; he had a medical history of atopic dermatitis. He was diagnosed with allergic conjunctivitis and treated with topical anti-allergic agents at a nearby clinic. However, since his symptoms did not improve and proliferative changes were observed in the conjunctiva, he was referred to Hokkaido University Hospital. Slit-lamp examination demonstrated marked lower eyelid swelling with an irregular massive conjunctival mass (Figure 2A). Magnetic resonance imaging revealed anterior orbital invasion of the tumor (Figure 2B). At this time, the patient withdrew informed consent and refused orbital exenteration, instead hoping to preserve the eyeball. Therefore, we decided to conduct local resection of the tumor tissues with lower eyelid reconstruction (Hughes flap) and used adjuvant topical IFN $\alpha-2 b$ eye drops. One month 

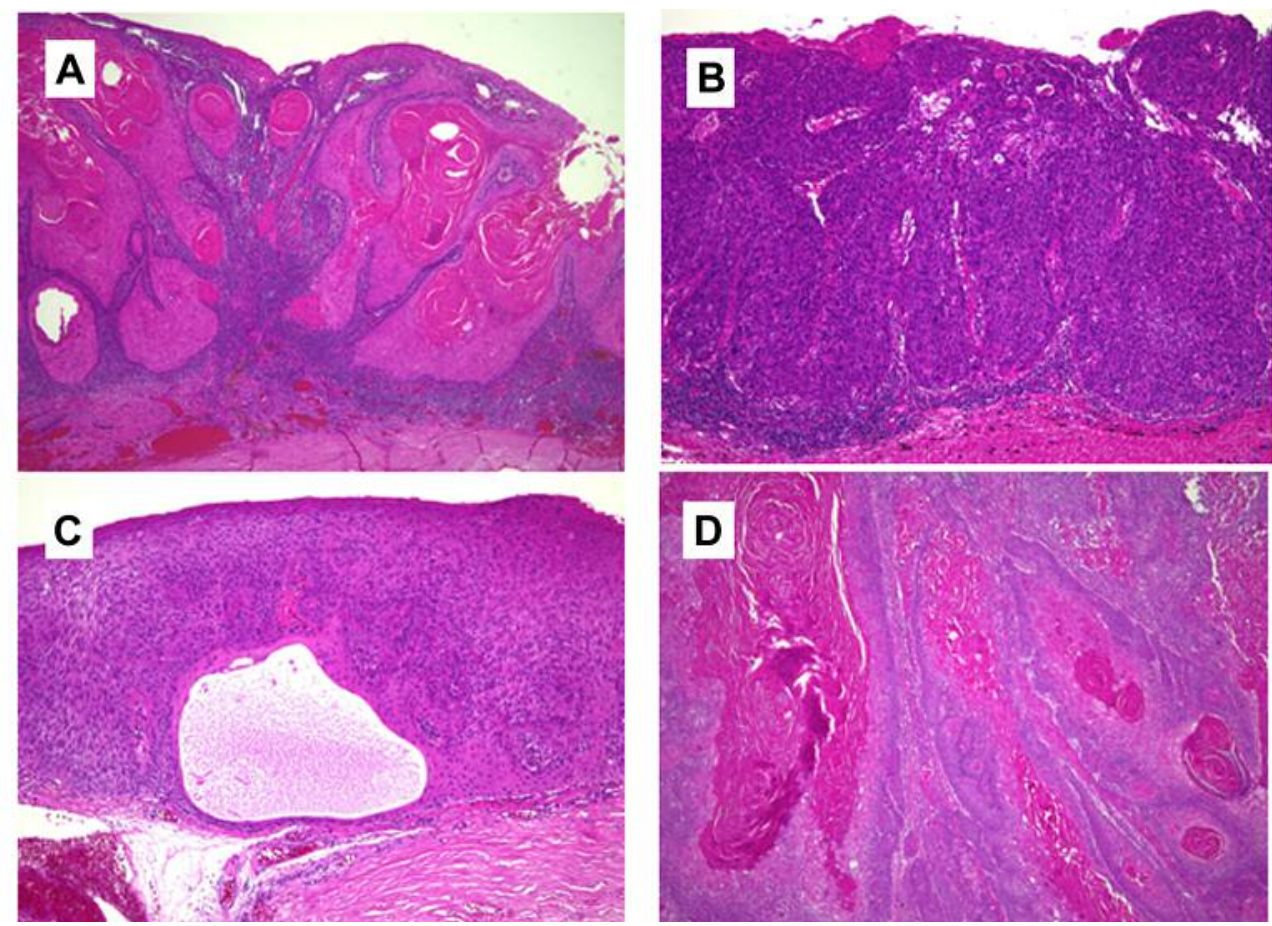

Figure 3. Histological findings of all the patients examined in this study. A: Case 1 showed invasive squamous cell carcinoma (SCC) forming cancer pearls. Hematoxylin-eosin staining, magnification $\times 40$. B: Case 2 demonstrated poorly differentiated SCC without marked keratinization. Hematoxylin-eosin staining, magnification $\times 40 . C$ : Case 3 demonstrated carcinoma in situ with full-thickness epithelial involvement by atypical cells. Hematoxylin-eosin staining, magnification $\times 40$. D: Case 4 showed highly invasive SCC with cancer pearl formation, indicating welldifferentiated SCC. Hematoxylin-eosin staining, magnification $\times 100$.

after resection, the reconstructed lower eyelid presented with favorable granulation-like tissue (Figure 2-C). The lower eyelid had undergone epithelialization, maintaining a smooth ocular surface (Figure 2D). The patient's best corrected visual acuity (BCVA) was 0.2 OD due to atopic posterior capsular cataract. There was no recurrence of the tumor in the ocular adnexa at 1 year after treatment. Since the biopsy was conducted from the lower bulbar conjunctiva and histology demonstrated no remaining tumor cells, he underwent cataract extraction with implantation of posterior chamber intraocular lens 18 months after the initial surgery (Figure 2E). BCVA recovered from 0.2 to 1.2 OD. Furthermore, the patient remains well without local recurrence, or intraocular or orbital invasion (Figure $2 \mathrm{~F}$ ) of the tumor 1.5 years after cataract surgery.

\section{Discussion}

Topical IFN- $\alpha 2$ b plays an important role in immunotherapy, immunoreduction, and immunoprevention (6). In our four cases with OSSN, histopathology of the resected tumor tissues suggested a positive surgical margin in the corneal side. Shields et al. showed that topical IFN $\alpha-2 b$ was effective for reducing the risk of recurrence after surgery (6). We have shown IFN $\alpha-2 b$ to be useful as an adjunctive treatment in conjunctival melanoma, thereby possibly promoting the host innate immune system (7). By adding IFN $\alpha$-2b eye drops, activation of the local immune system might have led to attack of potential residual tumor cells after tumor resection and prevented tumor recurrence in this study. These results suggest that topical IFN $\alpha-2 b$ is also effective in OSSN treatment as immunoprevention in this study.

As we show in a representative case, we for the first time successfully carried out cataract surgery in a case with conjunctival SCC arising in atopic dermatitis. OSSN can develop in the corneal tissues and invade intraocular tissues following intraocular surgery $(8,9)$, unless preoperative OSSN was appropriately managed before the surgery. Therefore, before intraocular surgery in patients having a history of OSSN, it is mandatory to ensure no tumor cells are left on the ocular surface based on repeated biopsies. Topical IFN $\alpha-2 b$ not only can save a patient's life, but also contribute to favorable QOV following cataract surgery.

In conclusion, Topical IFN $\alpha-2 b$ treatment contributed to suppression of tumor recurrence and improvement of quality of vision in patients after local resection of OSSN. 


\section{Conflicts of Interest}

The Authors declare no conflicts of interest.

\section{Authors' Contributions}

LK wrote the article. LK and SK collected the clinical and pathological data and evaluated those for patients in this study. KI and SI reviewed and critical revised the article.

\section{References}

1 Lee GA and Hirst LW: Ocular surface squamous neoplasia. Surv Ophthalmol 39: 429-450, 1995. PMID: 7660300.

2 Belardelli F, Ferrantini M, Proietti E and Kirkwood JM: Interferon-alpha in tumor immunity and immunotherapy. Cytokine Growth Factor Reviews 13: 119-134, 2002. PMID: 11900988

3 Endo H, Kase S, Suzuki Y and Kase M: Coincidence of inflamed conjunctival carcinoma in situ and primary pterygium. Case Rep Ophthalmol 27: 208-212, 2016. PMID: 28105011. DOI: $10.1159 / 000450824$

4 Poothullil AM and Colby KA: Topical medical therapies for ocular surface tumors. Semin Ophthalmol 21: 161-169, 2006 PMID: 16912014. DOI: 10.1080/08820530500351694

5 Venkateswaran N, Mercado C, Galor A and Karp CL: Comparison of topical 5-fluorouracil and interferon alfa-2b as primary treatment modalities for ocular surface squamous neoplasia. Am J Ophthalmol 199: 216-222, 2019. PMID: 3047124. DOI: 10.1016/j.ajo.2018.11.007
6 Shields CL, Ramasubramanian A, Mellen PL and Shields JA: Conjunctival squamous cell carcinoma arising in immunosuppressed patients (organ transplant, human immunodeficiency virus infection). Ophthalmology 118: 2133-2137, 2011. PMID: 21762990. DOI: 10.1016/j.ophtha.2011.04.001

7 Kikuchi I, Kase S, Ishijima K and Ishida S: Long-term followup of conjunctival melanoma treated with topical interferon alpha-2b eye drops as adjunctive therapy following surgical resection. Graefes Arch Clin Exp Ophthalmol 255: 2271-2276, 2017. PMID: 28752368. DOI: 10.1007/s00417-017-3754-2

8 Rootman DB, McGowan HD, Yücel YH, Pavlin CJ and Simpson ER: Intraocular extension of conjunctival invasive squamous cell carcinoma after pterygium surgery and cataract extraction. Eye and Contact Lens 38: 133-136, 2012. PMID: 22169876. DOI: 10.1097/ICL.0b013e318235c4d3.

9 Heinz C, Fanihagh F and Steuhl KP: Squamous cell carcinoma of the conjunctiva in patients with atopic eczema. Cornea 22: 135-137, 2003. PMID: 12605048.

10 Amin MB, Edge S, Greene F, Byrd DR, Brookland RK, Washington MK, Gershenwald JE, Compton CC, Hess KR, Sullivan DC, Jessup JM, Brierley JD, Gaspar LE, Schilsky, RL, Balch CM, Winchester DP, Asare EA, Madera M, Gress DM and Meyer LR: AJCC Cancer Staging Manual. Eighth Edition. Springer, 2017.
Received August 3, 2019

Revised September 10, 2019

Accepted September 20, 2019 